\title{
PROBLEM STRUCTURING IN THE ANALYTIC HIERARCHY PROCESS AND SOFT SYSTEMS METHODOLOGY
}

\author{
Doncho Petkov and OIga Mihova-Petkova \\ University of Natal, Private bag X01, Pietermaritzburg 3209, South Africa \\ Technikon Natal, P.O.B. 11078, Pietermaritzburg 3206, South Africa \\ petkov@comp.ump.ac.za/olga@pmbl.ntch.ac.za
}

\begin{abstract}
There have been some attempts by Saaty and Keams to show the links between systems ideas and the Analytic Hierarchy Process (AHP) especially with regard to the issue of problem complexity. It must be noted that their analysis is mainly with reference to a more traditional systems approach known in the past 15 years as hard systems thinking. Meanwhile in the last decade we have observed a growing maturity of a wider understanding of the systems field characterised in particular by the growing popularity of Soft Systems Methodology(SSM) by $P$. Checkland. Thus a question arises where does AHP stand with regard to SSM? The purpose of this research is to determine what are the similarities and differences between AHP and SSM with respect to problem formulation or problem structuring.
\end{abstract}

SSM is classified usually as a representative of a new, alternative or soft operations research stream of thought. Soft Operational Research or Problem Structuring Methods (PSM) seek a demarcation from the Analytic Hierarchy Process according to J. Rosenhead because of their transparency of method, restricted mathematisation and their focus on supporting judgement rather than representing it.

Dyer and Forman indicate that the Analytic Hierarchy Process focuses on the choice phase of decision making. It seems that such a statement makes unnecessarily narrow the scope of AHP. As long ago as 1982 Arbel and Tong have applied AHP for the generation of options in decision analysis problems. Their procedures apply both to the Intelligence and the Design phases of Simon's model of decision making. It may be noted however that the creativity processes in the intelligence and the design phase seem to be supported in a better way by brainstorming and some of the Problem Structuring Techniques. For example with respect to the stakeholder identification issue one may be apply SAST by Mason and Mitroff.

SSM is aiming more at supporting judgements rather than representing them. The expression of the problem situation is known as "rich pictures" which are not structured in a particular way. In our opinion AHP is stronger than Sof Systems Methodology with respect to providing means for making decisions since it not only provides means for supporting judgements but also for measuring them which is necessary for prioritising the issues in the intelligence and design stages of decision making.

SSM does not claim to be goal oriented. It is rather hermeneutic. According to Checkland organisations are studied with SSM from a hermeneutic stance. On the other hand AHP is a multicriteria decision support technique, paying special attention to goals and objectives. It can be noted that decision makers usually get a deeper insight into their problem through the formulation and exploration of an AHP model and especially through "What-if" sensitivity analysis which allows us to claim that AHP has both normative and interpretive features.

AHP is systemic because of its ability to include both quantitative and qualitative variables associated with a problem, the fact that a problem is structured in a hierarchy which has been identified in 1962 by H.Simon as a suitable way to handle complexity in systems and because of its ability to incorporate both objective and subjective information about organisational processes, design, culture and politics. On the other hand AHP cannot be used as a single systems methodology mainly for the fact that problem structuring is not formalised and there are better techniques for the Intelligence stage in Simon's model of decision making. That should not be viewed as a rejection of AHP as a systems science tool. On the contrary, it is our opinion that on the basis of critical systems thinking there is a common ground for the complementarist use of AHP with other methodologies. Taking into account the positive features of the two approaches we may come to the conclusion that there is a tremendous scope for new and fruitful combined applications of AHP with SSM. 